\title{
Associação de técnicas de clareamento em dentes não vitais: relato de caso
}

\author{
Association of teeth whitening techniques on non-vital teeth: case report
}

Asociación de técnicas de blanqueamiento dental en dientes no vitales: relato de caso

Heloisa França de Araujo ${ }^{1 *}$, Héberte de Santana Arruda1 ${ }^{1}$, Mariana Alves Lemos ${ }^{2}$, Amanda Maciel do Prado', Eduardo Borges da Costa Leite ${ }^{3}$, Marcos Antonio Japiassú Resende Montes¹.

\section{RESUMO}

Objetivo: Descrever um relato de caso em que o clareamento dental com associação das técnicas interna e externa foi o tratamento de escolha, abordando os materiais e os cuidados que devem ser tomados no momento da intervenção. Relato de caso: Paciente do sexo masculino procurou a clínica odontológica, pois se encontrava insatisfeito com a tonalidade do seu incisivo central, elemento 21. Inicialmente, foi realizada profilaxia com pasta à base de pedra-pomes e água, em escova de Robinson, de todas as superfícies dentais. Logo em seguida realizou-se a abertura da câmara pulpar, limpando-a completamente, removendo todo o material restaurador. Após o selamento cervical foram feitas três sessões de clareamento na técnica externa e a técnica "walking bleach". Considerações finais: A técnica de consultório foi eleita em virtude das necessidades do paciente associada com a eficácia e segurança do agente clareador empregado, amplamente difundida na literatura. Para que se tenha um resultado satisfatório no clareamento de dentes desvitalizados é necessário um diagnóstico preciso, um plano de tratamento adequado, correta decisão sobre as técnicas a serem empregadas, destreza e habilidade do cirurgião-dentista para aplicá-las e dessa forma concluir o caso com segurança e satisfação do paciente.

Palavras-chave: Clareamento dental, Clareadores, Estética dentária, Dente não vital.

\begin{abstract}
Objective: To describe a case report in which tooth whitening with the association of internal and external techniques was the treatment of choice, addressing the materials and care that must be taken at the time of the intervention. Case report: A male patient attended the dental clinic, as he was dissatisfied with the tone of his central incisor, element 21. Initially, prophylaxis was performed with pumice paste and water, using a Robinson brush, of all dental surfaces. Soon afterwards, the pulp chamber was opened, cleaning it completely, removing all the restorative material. After cervical sealing, three bleaching sessions were performed using the external technique and the walking bleach technique. Final considerations: The in-office technique was chosen due to the patient's needs associated with the efficacy and safety of the bleaching agent used, which is widely disseminated in the literature. In order to have a satisfactory result in whitening devitalized teeth, it is necessary to have an accurate diagnosis, an adequate treatment plan, correct decision on the techniques to be employed, the dentist's skill and ability to apply them and thus conclude the case with patient safety and satisfaction.
\end{abstract}

Key words: Tooth bleaching, Bleaching agents, Esthetics dental, Tooth non vital.

\footnotetext{
1 Universidade de Pernambuco (FOP/UPE), Camaragibe - PE. *E-mail: heloisaraujo24@gmail.com

2 Instituto de Medicina Integral Professor Fernando Figueira (IMIP), Recife - PE.

3 Universidade Federal de Pernambuco (UFPE), Recife - PE.
} 


\section{RESUMEN}

Objetivo: Informar de un caso clínico en que el blanqueamiento dental con la asociación de técnicas internas y externas fue el tratamiento de elección, abordando los materiales y el cuidado que deben tomarse en el momento de la intervención. Reporte de un caso: Un paciente masculino asistió a la clínica dental, ya que no estaba satisfecho con el tono de su incisivo central. Inicialmente, la profilaxis se realizó con pasta de piedra pómez y agua. usando un cepillo Robinson, de todas las superficies dentales. Después, se abrió la cámara de pulpa, limpiándola por completo, eliminando todo el material restaurador. Después del sellado cervical, se realizaron tres sesiones de blanqueamiento utilizando la técnica externa y "blanqueador ambulante". Consideraciones finales: La técnica de consultorio se eligió debido a las necesidades del paciente asociadas con la eficacia y seguridad del agente blanqueador utilizado, que está ampliamente difundido en la literatura. Para tener un resultado satisfactorio en el blanqueamiento de los dientes desvitalizados, es necesario contar con un diagnóstico preciso, un plan de tratamiento, decisión correcta sobre las técnicas a emplear, la habilidad y la capacidad del dentista para aplicarlas y así concluir el caso con seguridad y satisfacción del paciente.

Palabras clave: Blanqueamiento de dientes, Blanqueadores, Estética dental, Diente no vital.

\section{INTRODUÇÃO}

O escurecimento de dentes desvitalizados tem se apresentado como uma situação bastante recorrente na clínica diária (MONTEIRO RV, et al., 2018; SANTOS-JUNIOR AO, et al., 2018), principalmente em dentes anteriores onde é rapidamente percebido, gerando um desconforto e prejudicando a harmonia do sorriso, comprometendo assim, o bem-estar e a autoestima (NETTO PCP, 2013). Essa alteração cromática pode ocorrer por diversas razões como hemorragia decorrente de trauma, calcificações pulpares, abordagem terapêutica inadequada, espaço de tempo entre o traumatismo e o atendimento odontológico, longevidade da restauração provisória após o tratamento endodôntico e excesso de materiais obturadores como cones de guta-percha e cimentos endodônticos no interior da câmara pulpar (SANTOS-JUNIOR AO, et al., 2018).

Frente a este desafio, o cirurgião-dentista pode abordar algumas estratégias de planejamento para alcançar as expectativas do paciente. O tratamento deve ser realizado através de uma abordagem multidisciplinar, levando não só em consideração o design do sorriso, como também a inter-relação entre todas as estruturas orais de suporte, incluindo músculos, ossos, articulações, tecidos gengivais e oclusão. Assim, pode-se obter um resultado bem-sucedido, saudável e funcional (ZAVANELLI AC, et al., 2017).

As abordagens de clareamento dental são classificadas didaticamente em grupos de acordo com o local de execução, em externo e interno; com o tempo de permanência do agente clareador em imediata ou termocatalítica, mediata ou walking bleach e mista (LUCENA MTL, et al., 2015; SANTOS-JUNIOR AO, et al., 2018). O profissional também pode associar mais de um método a fim de obter o resultado estético almejado (LUCENA MTL, et al., 2015; MENDONÇA NJ, et al., 2018).

O clareamento dental externo apresenta-se como um procedimento consolidado no meio odontológico. Possui diferentes abordagens que variam de acordo com o desejo do paciente e o plano de tratamento delineado pelo profissional. A técnica do clareamento caseiro se mostra como uma alternativa mais conservadora, pois utiliza um gel clareador mais fraco e moldeiras plásticas (acetato ou silicone) confeccionadas para uso pelo próprio paciente, supervisionado pelo dentista. $\mathrm{Na}$ técnica de consultório, a concentração do gel clareador é aumentada e o produto é aplicado exclusivamente pelo profissional. Existem outras abordagens disponíveis no mercado como as fitas clareadoras, porém há divergências na literatura sobre sua eficácia (BISPO LB, 2018). Já no clareamento dental interno, o agente clareador é inserido na câmara pulpar como um curativo de demora (MENDONÇA NJ, et al., 2018).

A utilização de substâncias clareadoras no interior da câmara pulpar mostra-se uma manobra conservadora frente a alterações cromáticas de dentes desvitalizados (CARDOSO RM, et al., 2011). Na técnica imediata ou termocatalítica o profissional aplica o agente clareador no interior da câmara pulpar e sobre a superfície vestibular do elemento, sendo o resultado clareador bem evidente e aceito pelo paciente já nas primeiras sessões, seja ele fotoassistido ou não (LUCENA MTL, et al., 2015; MILESKI T, et al., 2018). 
Já a mediata ou walking bleach é baseada no preenchimento da cavidade, o paciente permanece com o produto no interior da câmara pulpar em forma de curativo (CARDOSO RM, et al., 2011). Já a técnica mista é uma associação das duas técnicas supracitadas (LUCENA MTL, et al., 2015; MILESKI T, et al., 2018). É de fundamental importância observar o comportamento do dente frente aos procedimentos, pois, constatada ausência de reversibilidade cromática, o profissional pode optar por soluções restauradoras ou protéticas (CARDOSO RM, et al., 2011).

Um dos agentes clareadores mais utilizados e seguros para clarear pigmentação de origem endógena é o Perborato de Sódio (SANTOS-JUNIOR AO, et al., 2018). O mesmo se dissocia no peróxido de hidrogênio (liberação de oxigênio) em meio alcalino, mas em contrapartida, apresenta menor velocidade de liberação de oxigênio (BOAVENTURA JMC, et al., 2012). O principal benefício da utilização desse material está na baixa concentração de peróxido de hidrogênio liberada, bem como na presença de ureia que é um composto que regula o $\mathrm{pH}$ intracoronário durante o processo clareador. Quando possui carbopol este faz com que a liberação de oxigênio seja retardada, atuando com menor intensidade, porém por um maior período de tempo (LUCENA MTL, et al., 2015).

Diante disso, o objetivo deste artigo é apresentar um relato de clareamento dental utilizando uma associação de técnicas, abordando os materiais e os cuidados que devem ser tomados no momento da intervenção para que se possa obter o sucesso clínico desejado.

\section{RELATO DE CASO}

Paciente de 25 anos, do sexo masculino, procurou a Clínica do Curso de Especialização em Dentística, pois se encontrava insatisfeito com a tonalidade do seu incisivo central, elemento 21 , em relação aos outros elementos, o que o deixava incomodado ao sorrir (Figura 1). Durante a avaliação confirmou-se a presença de tudo que foi relatado pelo paciente e constatou-se uma desarmonia entre os incisivos centrais decorrente da mesialização do elemento em questão.

Figura 1 - Registro fotográfico da condição clínica inicial do paciente.

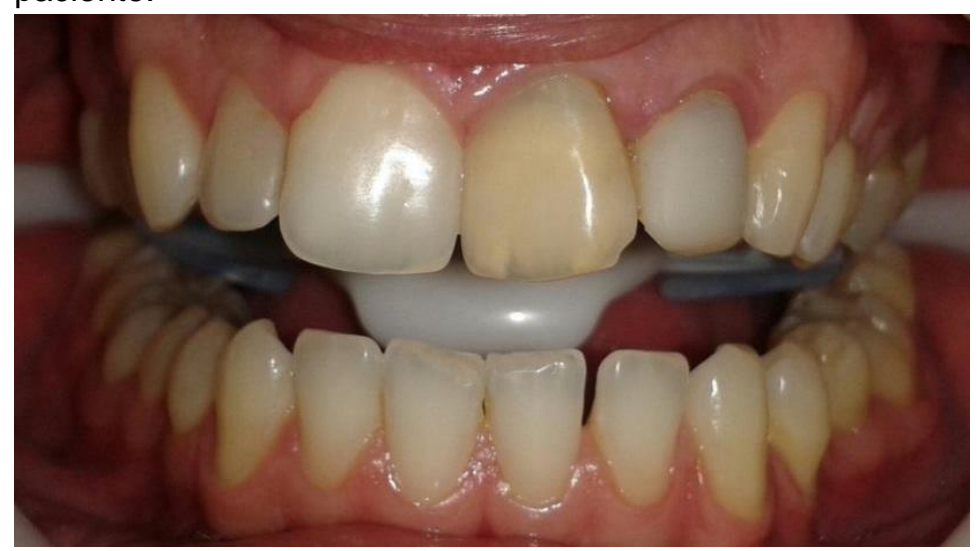

Fonte: Leite EBC, et al., 2019.

Após realização de exame clínico, radiográfico e fotográfico, certificou-se que o tratamento endodôntico estava adequado e elaborou-se um plano de tratamento que consistiu em fazer uma associação de técnicas, o clareamento interno ao clareamento de consultório, visando alcançar um resultado mais eficaz.

Inicialmente, foi realizada uma profilaxia com pasta à base de pedra-pomes e água, em escova de Robinson, de todas as superfícies dentais. Logo em seguida realizou-se a abertura da câmara pulpar, limpando-a completamente, removendo todo o material restaurador (Figura 2A). O próximo passo, após a realização do isolamento absoluto dos incisivos superiores, foi fazer a mensuração vestibular para definir o comprimento do canal a ser desobturado, calculando $3 \mathrm{~mm}$ além da margem cervical. Estabelecido o comprimento, selecionou-se uma broca Largo compatível com as dimensões do canal e retirou-se o material obturador do conduto radicular conforme previsto na medição (Figura 2B). 
Figura 2 - A) Abertura coronária e B) Remoção do material obturador.

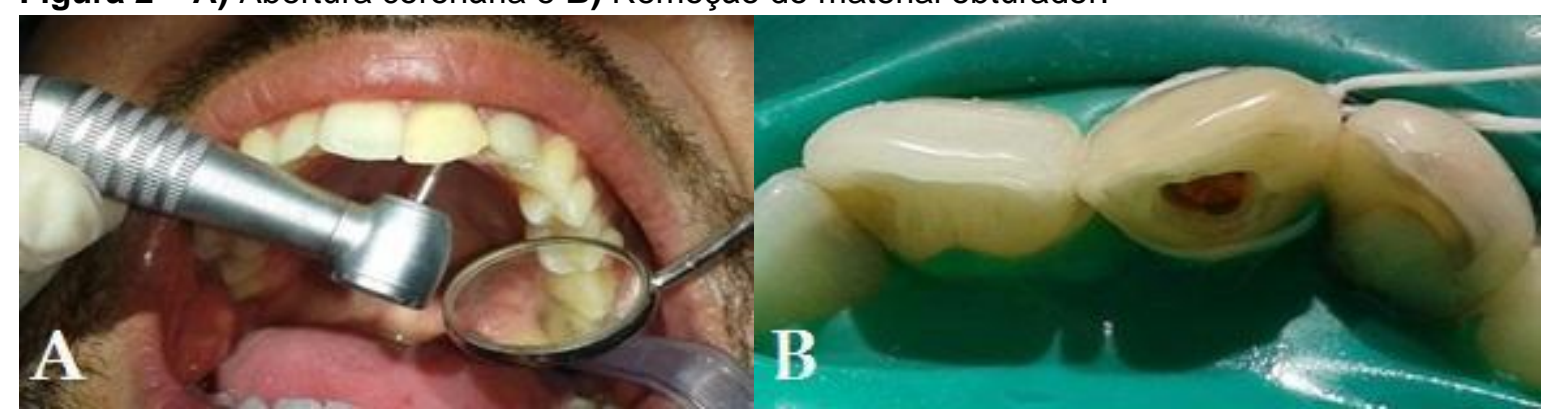

Fonte: Leite EBC, et al., 2019.

Posterior ao condicionamento ácido da cavidade com o ácido fosfórico 37\% (Condac-FGM) e aplicação do sistema adesivo, fez-se um selamento cervical de aproximadamente $3 \mathrm{~mm}$ de espessura utilizando o cimento resinoso Allcem Core (FGM). O selamento cervical tem o objetivo de evitar a passagem de clareador para o periodonto, sendo esta uma etapa crítica do processo e deve prover um selamento perfeito (Figura 3). Em seguida, preparou-se a pasta de Whiteness Perborato, numa superfície de plástico, misturando duas porções rasas de pó com uma gota de solução de peróxido de hidrogênio e aplicou-se o clareador no interior da câmara pulpar até preencher dois terços do seu volume. Em seguida selou-se a cavidade com ionômero de vidro (Maxxion R - FGM) e verificou-se a oclusão do paciente em movimentos de protrusão e lateralidade, pois o contato prematuro pode fraturar a restauração provisória e/ou dente.

Figura 3 - Selamento cervical utilizando o cimento Allcem Core (FGM).

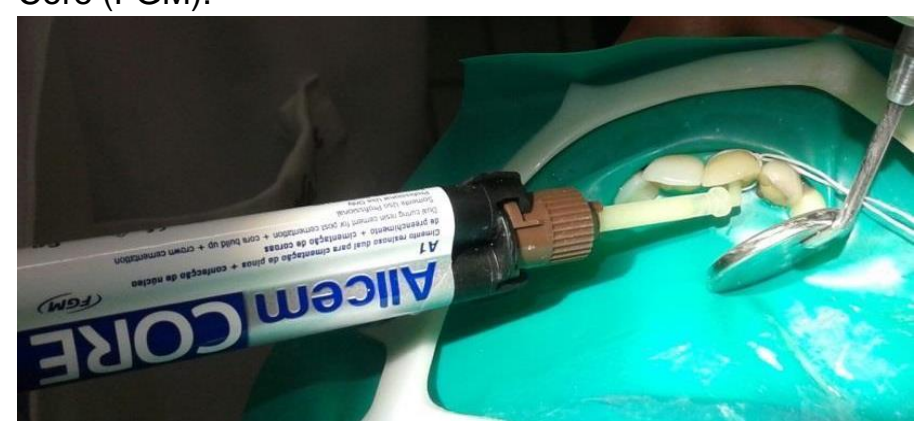

Fonte: Leite EBC, et al., 2019.

Em seguida, promoveu-se a aplicação do agente clareador (Whiteness HP maxx 35\%) o qual agiu na estrutura dentária externa por 40 minutos (Figura 4). Ao final da sessão clínica, o gel clareador foi removido utilizando uma cânula aspiradora, o dente foi lavado com água, removeu-se o isolamento e o paciente foi liberado com as devidas recomendações. Após quatro dias, ele retornou a clínica para reavaliação do procedimento e já demonstrava certo grau de satisfação com a saturação que o dente atingiu (Figura 5).

Figura 4 - Aplicação do agente clareador externo

Whiteness HP Maxx 35\%

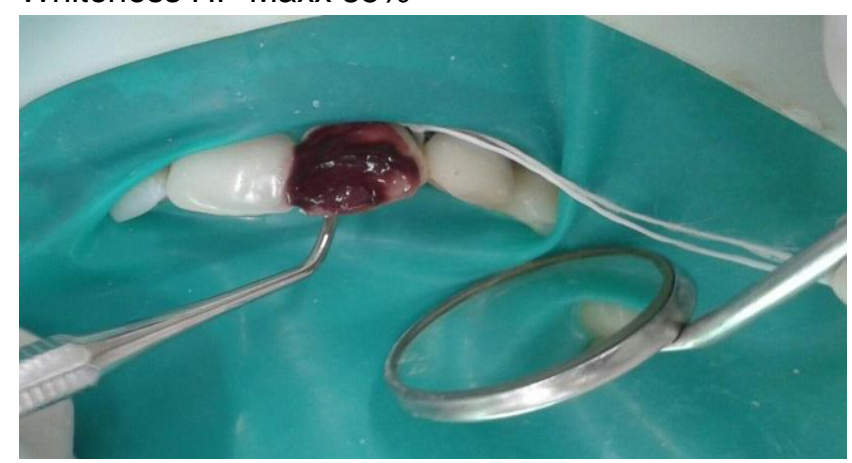

Fonte: Leite EBC, et al., 2019. 
Figura 5 - Registro fotográfico após o final da 1롤 sessão.

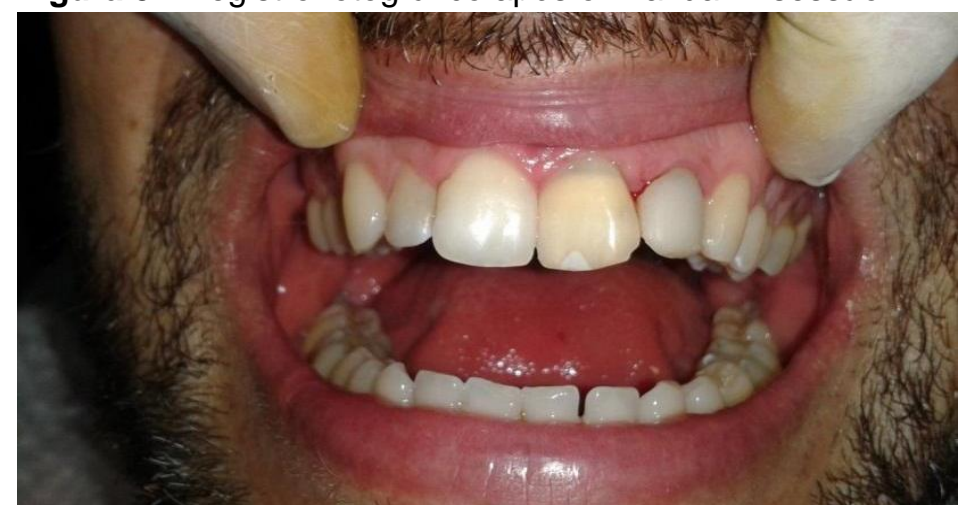

Fonte: Leite EBC, et al., 2019.

Desta forma, removeu-se o agente clareador do interior da câmara pulpar e aguardou-se sete dias para repetição do procedimento (Figura $6 \mathrm{~A}$ ). Foram realizadas três sessões clínicas com intervalos de uma semana entre as mesmas, obtendo-se uma coloração final satisfatória ao paciente (Figura 6B). Após o término do tratamento clareador, aguardou-se uma semana para realização da restauração definitiva, conforme recomendações do fabricante.

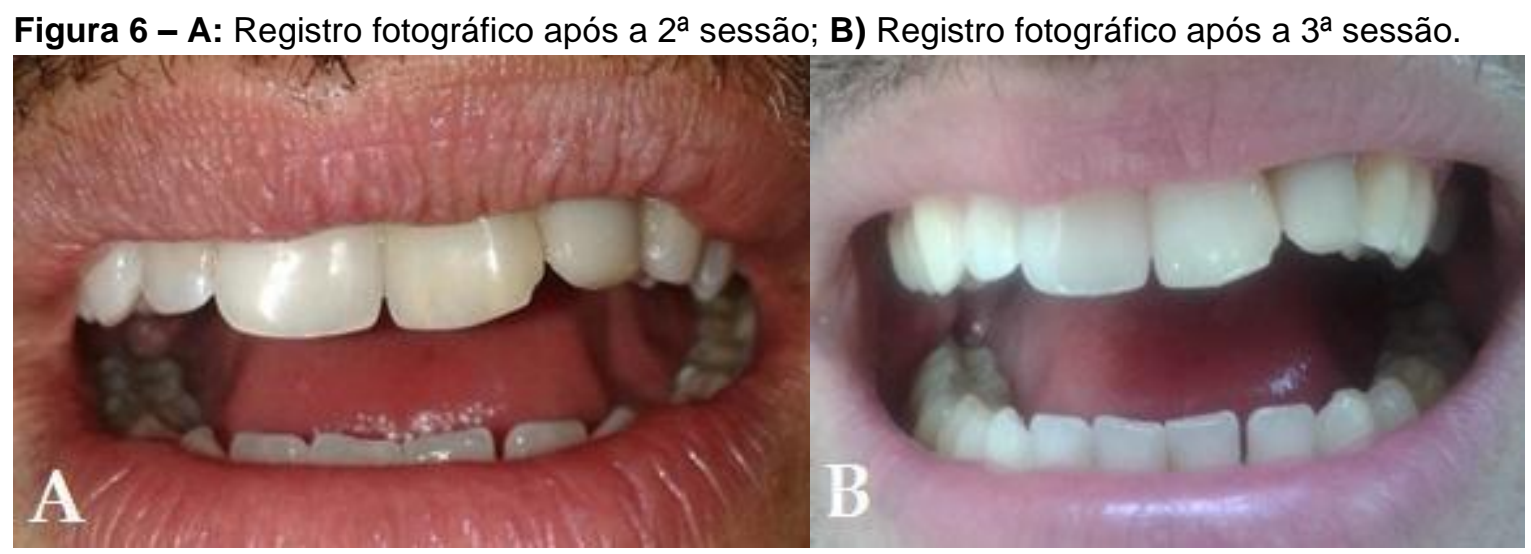

Fonte: Leite EBC, et al., 2019.

\section{DISCUSSÃO}

A alteração de cor dos dentes é um dos principais motivos de insatisfação com a estética e a harmonia do sorriso, levando à busca por um tratamento que restabeleça as características naturais (LUCENA MTL, et al., 2015). No caso clínico descrito, foi diagnosticado que o escurecimento ocorreu devido a presença de excesso de material obturador na câmara pulpar do elemento dentário, corroborando com o que já preconizava Baratieri LN, et al. (2001), como uma das principais razões para o escurecimento de dentes desvitalizados.

O clareamento dental externo é relatado na literatura como uma boa alternativa frente a técnicas restauradoras como coroas e facetas por ser um procedimento seguro, de fácil execução, não-invasivo e mais acessível financeiramente aos pacientes quando comparado a procedimentos protéticos (MENDES BMS, et al., 2011; TOLEDO FL, et al., 2009).

A terapia clareadora pode ser conduzida de uma maneira mais amena, empregando a técnica caseira com o peróxido de carbamida ou de forma mais intensa na chamada técnica "in-office" ou de consultório, que faz uso de substâncias mais concentradas. A técnica de consultório foi eleita em virtude das necessidades do paciente associada com a eficácia e segurança do agente clareador empregado, que é amplamente difundida na literatura. O padrão de saturação inicial bem como a idade do paciente auxiliou na escolha da técnica empregada, pois, tomando as devidas precauções, atingiu-se um resultado de excelência sem danos estéticos ou efeitos colaterais indesejados (ALMEIDA LCAG e BRISCO ALF, et al., 2009; NASCIMENTO JPN, 2019). 
Os achados de Yui KCK, et al. (2008), sugerem que a técnica mista de clareamento é utilizada em casos de dentes escurecidos com difícil resolução, pois consegue potencializar o efeito clareador e obter resultados mais eficazes. A associação das técnicas de clareamento apresentou resultados satisfatórios e estão de acordo com que foi achado por Edhardt MCG, et al. (2003), que avaliou em seu estudo a eficácia de diversas técnicas clareadoras, e observaram que o uso do perborato de sódio em combinação com o peróxido de hidrogênio promoveu um efeito clareador maior e mais rápido. O mesmo em contato com a água se decompõe em metaborato de sódio, oxigênio e peróxido de hidrogênio. O peróxido de hidrogênio libera oxigênio ativando o processo clareador (ARAUJO JLS, et al., 2015).

Pesquisas afirmam que o perborato de sódio é eficaz quando usado com diferentes veículos além da água, como por exemplo, peróxido de hidrogênio e géis de clorexidina, embora alguns autores defendam que a associação do perborato de sódio ao peróxido de hidrogênio, como empregado neste caso clínico, apresenta resultados mais rápidos, expressivos e satisfatórios (SAMPAIO MD, et al., 2010; MACIEL KBL, et al., 2019).

Contudo, um fator importante a ser considerado foi destacado por Lado EA et al. (1983), quando indicou em seus registros, e todos os autores preconizam desde então, a necessidade de um adequado selamento cervical visando minimizar os riscos de uma possível passagem do agente clareador para os tecidos periodontais. Este selamento caracterizaria de fato uma barreira, sendo o mesmo confeccionado em uma camada de 1 a $3 \mathrm{~mm}$ de um material selador (Cimento Resinoso, Resina Flow, lonômero de Vidro Restaurador, etc...) (LUCENA MTL, et al., 2015).

Essa técnica apresenta resultados esteticamente satisfatórios, no entanto, está relacionado ao risco de reabsorção radicular externa (RCE), caso as etapas do tratamento não sejam realizadas corretamente ou, a seleção do caso não seja feita de forma criteriosa (BROWN BD, 1965; HARRINGTON GW e NATKIN E, 1979; FRIEDMAN S, 1997). Apesar disso, dentre as técnicas de clareamento interno a walking bleach mostrou oferecer menores riscos de RCE (LUCENA MTL, et al., 2015).

Uma das limitações do clareamento de dentes tratados endodonticamente é a recidiva da cor obtida inicialmente (ATTIN T, et al., 2003). Os resultados iniciais, apesar de muito satisfatórios na maioria dos casos, não podem ser considerados permanentes. Essa recidiva pode ser devido a uma redução química dos produtos gerados durante a ação dos agentes clareadores, infiltração marginal de restaurações, produtos químicos ou bacterianos e trauma dental. (WATERHOUSE PJ e NUNN JH, 1996). Sendo assim, o acompanhamento periódico se mostra fundamental para manutenção do resultado obtido.

A associação entre o clareamento de consultório e o walking bleach é uma técnica muito utilizada e mostra resultados rápidos e satisfatórios, porém, necessita do conhecimento e do domínio das técnicas empregadas por parte do cirurgião-dentista para obter um resultado de excelência. Diante disso, a escolha dessa técnica para esse paciente permitiu uma melhora significante na estética do sorriso.

Para que se obtenha o sucesso almejado no clareamento de dentes escurecidos é essencial a realização de um correto diagnóstico e planejamento do caso, assim como a escolha de técnicas conservadoras e materiais clareadores seguros e eficazes. Além disso, a correta execução da técnica escolhida é indispensável para garantir a segurança do procedimento realizado.

\section{REFERÊNCIAS}

1. ALMEIDA LCAG, BRISCO ALF. Sucesso Clareador com a Tradicional Técnica Caseira. Revista FGMNews, $2009 ; 11$ : 50-54.

2. ARAÚJO JLS, et al. Técnicas de clareamento dental - revisão de literatura. Revista Pró-univerSUS, 2015; 6(3): 35-37.

3. ATTIN T, et al. Review of the current status of tooth whitening with the walking bleach technique. International endodontic journal, 2003; 36(5): 313-329.

4. BARATIERI LN, et al. Odontologia Restauradora: Fundamentos e Possibilidades. São Paulo: Santos, 2001; 739p.

5. BISPO LB. Clareadores dentários contemporâneos: tópicos. Rev. Odontol. Univ. Cid. São Paulo, 2018; 30(2): $177-89$.

6. BOAVENTURA JMC, et al. Clareamento para dentes despolpados: revisão de literatura e considerações. Rev. Odontol. Univ. Cid. São Paulo, 2012; 24(2): 114-122.

7. BROWN BD. Factors influencing successful bleaching of the discolored root-filled tooth. Oral Surg. Oral Med. Oral Pathol, 1965; 20(2): 238-244. 
8. CARDOSO RM, et al. Clareamento interno: uma alternativa para discromia de dentes tratados endodonticamente. Odontol. Clín.-Cient. (Online), 2011; 10(2): 177-180.

9. ERHARDT MCG, et al. Clareamento Dental interno. RGO, 2003; 51(1): 23-9.

10. FRIEDMAN S. Internal bleaching: long-term outcomes and complications. J. Am. Dent. Assoc, 1997; 128: 51S-55S.

11. HARRINGTON GW, NATKIN E. External resorption associated with bleaching of pulpless teeth. J. Endod, 1979; 5(11): 344-348.

12. LADO EA, et al. Cervical resorption in bleached teeth. Oral Surg. Oral Med. Oral Pathol, 1983; 55(1): 78-80.

13. LUCENA MTL, et al. Clareamento interno em dentes desvitalizados com a técnica walking bleach - relato de caso. Uningá Review, 2015; 24(1): 33-39.

14. MACIEL KBL, et al. Clareamento em um dente desvitalizado: relato de caso. Revista Eletrônica Acervo Saúde, 2019; 18: $1-6$.

15. MENDES BMS, et al. Clareamento externo de dente não vital. Revista Saúde UNG-SER, 2011; 5(2): 46-55.

16. MENDONÇA NJ, et al. Tratamento de reabsorção radicular externa associada ao clareamento dental endógeno: relato de caso. Psicologia E Saúde Em Debate, 2018; 4(1): 101-101.

17. MILESKI T, et al. Clareamento interno em dente traumatizado: relato de caso clínico. Revista UNINGÁ, 2018; 55(2): 24-32.

18. MONTEIRO RV, et al. Técnica de clareamento caseiro modificada para dente não vital: relato de caso. RSC online, 2018; 7(1): 86-93.

19. NASCIMENTO JPN. Avaliação da eficácia entre os métodos de clareamento dental caseiro x de consultório - Revisão de Literatura. Dissertação (Graduação em Odontologia) - Centro Universitário do Planalto Central Apparecido dos Santos, Gama-DF, 2018: 9 p.

20. NETTO PCP. Clareamento de dentes desvitalizados: relato de caso clínico. Dissertação (Graduação em Odontologia) - Departamento de Odontologia. Universidade Estadual de Londrina, Londrina, 2013; 32 p.

21. SAMPAIO MD, et al. Análise espectrofotométrica do clareamento dental interno. RGO, Rev. gaúch. odontol. (Online), 2010; 58(3): 363-368.

22. SANTOS-JÚNIOR AO, et al. Recuperação da coloração de dentes tratados endodonticamente através das técnicas clareadoras imediata e mista. Salusvita, 2018; 37( 1): 77-91.

23. TOLEDO FL, et al. Clareamento interno e externo em dentes despolpados - caso clínico. Revista da Faculdade de Odontologia de Lins, 2009; 21(2): 59-64.

24. WATERHOUSE PJ, NUNN JH. Intracoronal bleaching of nonvital teeth in children and adolescents: interim results. Quintessence Int, 1996; 27(7): 447-452.

25. YUI KCK, et al. Ex vivo evaluation of the effectiveness of bleaching agents onthe shade alteration blood-stained teeth. Int Endod J, 2008; 41(6): 485-92.

26. ZAVANELLI AC, et al. Previsibilidade do tratamento estético com lentes de contato cerâmicas. Archives of Health Investigation, 2017; 6(12): 598-603. 